University of Nebraska - Lincoln

DigitalCommons@University of Nebraska - Lincoln

Robert Katz Publications

Research Papers in Physics and Astronomy

January 1993

\title{
A Track Physics Retrospective
}

Robert Katz

University of Nebraska-Lincoln, rkatz2@unl.edu

Follow this and additional works at: https://digitalcommons.unl.edu/physicskatz

Part of the Physics Commons

Katz, Robert, "A Track Physics Retrospective" (1993). Robert Katz Publications. 117.

https://digitalcommons.unl.edu/physicskatz/117

This Article is brought to you for free and open access by the Research Papers in Physics and Astronomy at DigitalCommons@University of Nebraska - Lincoln. It has been accepted for inclusion in Robert Katz Publications by an authorized administrator of DigitalCommons@University of Nebraska - Lincoln. 
Published in Radiation Protection Dosimetry 47 (1993), 65-68. Copyright (C) 1993 Nuclear Technology Publishing; published by Oxford University Press. Used by permission. http://rpd.oxfordjournals.org/

\title{
A Track Physics Retrospective
}

\author{
Robert Katz \\ University of Nebraska-Lincoln \\ Lincoln, NE 68588-0111, USA
}

\begin{abstract}
Many condensed matter dosimeters and detectors respond to the perspectives of track physics, a parametric model which relates their response to gamma rays to their response to heavy ions through the radial distribution of dose about a heavy ion's path. Most dosimeters are treated as 1-hit detectors, a classification which includes emulsions, scintillators, TLDs, the Fricke dosimeter, alanine, and some biological substances: enzymes and Eschericia coli, among others. We have discovered many hit detectors. An extended model describes the killing, transformation, and mutation of biological cells after gamma and heavy ion irradiation. A detector is treated as a black box, in which the only relevant information is the nature of the incident radiation and the output response, although, at times, it is convenient to imagine some simple structure. Because the model is parametric it can be global, applying to many detectors. Once a set of numerical parameters is extracted from experimental findings, the equations of the model predict response to all radiation fields whose photon-particle energy spectrum is known. Finally, parameters of the model serve as a test of the validity of mechanistic theories, for these parameters summarize detector response to all ionizing radiations.
\end{abstract}

\section{Introduction}

Track physics was created in 1965 to characterize the width of heavy ion tracks in electron sensitive nuclear emulsions exposed to cosmic rays. [1] There the radial distribution of dose from delta rays was identified as the central criterion determining track structure. A few years later, in 1968, a parallel criterion was suggested for the formation of etchable tracks in dielectrics. [2] This criterion, of minimal dose at a minimal distance, has only recently been examined critically and confirmed experimentally [3] as the basis of "etching rate." In 1967 the application of track concepts to radiobiological data was undertaken, to seek for the basis of RBE, the relative biological effectiveness of energetic heavy ions. It was asserted that the response of the targets comprising a detector to delta rays must be identical to the response of these targets to the secondary electrons from gamma rays, in the belief that these targets could not distinguish the source of the electrons that traversed them. Data were found on the inactivation cross sections of dry enzymes and viruses by energetic heavy ions. It was inferred that exponential survival curves would also be obtained from gamma irradiation, as consistent with the cumulative Poisson distribution for 1-ormore-hit systems, and a radial distribution of inactivation probability from the gamma ray response and the radial dose distribution was calculated. The radial integral of the inactivation probability was taken to be the theoretical cross section. [4] This was our first venture into the concept of the 1-hit detector, later to be improved through a more sophisticated calculation of the radial dose distribution. [5] Here, for the first time, a connection was established between the response of a detector to gamma rays and its response to energetic heavy ions. This was a revolutionary concept, and changed forever the way people thought about the response of detectors to HZE particles. It prompted a series of measurements and calculations of the radial distribution of dose [6], which continue to the present day. Where the words "track structure" were virtually unheard before our work, these words now permeate all discussions of the effects of HZE particles. Other concepts through which HZE response are discussed, like LET, restricted LET, thermal spike, ion explosion spike, associated volume, track core, track penumbra, $\mathrm{Y}$, and $\mathrm{Z}$ distributions are heard with diminishing frequency.

The cross section for 1-hit detectors depended much more on the $X$ ray response and on the radial dose distribution than on the size of the target. $z / \beta$ was found to be a more appropriate descriptor than LET because delta ray production dominated the observed response, though in the "thindown" region at track end, track properties correlated better with $\beta$ because of kinematic restrictions on the energy of delta rays. In calculated plots of cross sections against LET, the effect of thindown appeared as branched "hooks" at highest LET, a prediction which was later fulfilled in radiobiology and for several physical detectors. A track structure interpretation is demanded whenever detector response is multiple valued at the same LET, from different ion bombardments, most evident in these "hooks." Such variations have now even been observed in experiments in which the properties of materials are altered by intense bombardments with energetic heavy ions. [7] 


\section{Radiation Detectors and Dosimeters}

\section{Emulsion}

The concept of a 1-hit detector, first developed in the study of the cross sections for dry enzymes and viruses, clarified the description of particle tracks in electron sensitive emulsion. Now it was possible to calculate the radial distribution of developable grains. From this, the "grain count" was foundthe number of developed grains per $100 \mu \mathrm{m}$, and the track opacity as measured in a microdensitometer, as a function of the characteristic dose of $\gamma$ rays, a fitted parameter. Much published data [8] yielded to these descriptions. A "grain-count regime" was identified and a "track width regime," where tracks appeared like a "hairy rope," for these had different response characteristics. For example, the "hooks" could only be seen in the "track width regime," while the "Bragg peak" was only approximated in detector response in the "grain count regime." This model of particle tracks was applied at Lund for their emulsion based cosmic ray studies. [9] To simulate the properties of desensitized emulsion, the response to $r$ rays was assumed to have the form of the cumulative Poisson C-or-more-hit distribution. Computer simulations of such tracks could be fitted to tracks of Ar ions in emulsions which were desensitized in manufacture or were developed in such a way as to discriminate against small latent image clusters. [10] In these materials, single electron tracks were invisible. The tracks of heavy ions strongly discriminated against low LET particles in favor of high LET particles. Hittedness as high as 8 was achieved. These tracks resembled inferences of particle tracks in a matrix of biological cells where the RBE was greater than 1 . The intention of exploring these materials as high LET dosimeters was frustrated by technical problems in emulsion manufacture.

\section{Detectors lacking granular structure}

The track structure model is based on the probability of activation of granular targets. Yet many detectors whose response clearly follows the pattern of a 1-hit detector have no overt granular structure. Calculational procedures then depend on the desired endpoint. One can simply transfer the gamma ray response characteristic to the radial dose distribution to find the radial distribution of effect, the "size" of the affected region. Alternatively, in these cases one can assume that the interaction of secondary electrons with the medium is restricted to distances through which interaction products or en- ergy can migrate by diffusion to an appropriate site. In $\mathrm{NaI}(\mathrm{Tl})$ the site was taken to be the thallous ion. [11] The size of the relevant region was based on data for response relative to $\mathrm{Tl}$ concentration. In the Fricke dosimeter the site was taken to be the ferrous ion, and $\mathrm{OH}$ radicals formed in water were taken to diffuse to that site through a limited distance [12], a fitting parameter. In some cases a simple "pointtarget" calculation of the relative effectiveness was made in which one only required a single parameter, the characteristic dose from which a cross section could be calculated. The model describes such varied dosimeters and processes as lyoluminescence [13], alanine [14], F-center formation in LiF [15], coloration of dye-cyanide films [16], and so on. From a mechanistic point of view this generality is startling. But the unifying issue is that the observed signal is created by secondary electrons, and that for 1-hit detectors a single electron transit through a sensitive site is adequate to activate it.

For I-hit detectors the cross section $\boldsymbol{\sigma}$ is related to other measures of radiation response. Thus the number of activated targets per unit path length is $\sigma N$, where $N$ is the number density of targets, and the $G$ value is $\sigma N / L$, where $L$ is the LET or stopping power. The linear density of activated targets is the "grain count" in emulsion and is proportional to pulse height in a scintillation counter. The $G$ value is measured in a Fricke dosimeter. The number of activated sites in a crystal is proportional to the TLD response. In 1-hit detectors all these derive from knowledge of the cross section. If we cannot be certain of the number density of sensitive targets, only relative response can be calculated.

\section{Biological 1-hit detectors}

Single strand breaks in DNA have long been identified as biological 1-hit detectors. So also is the inactivation of dry enzymes and viruses. These, our first 1-hit detectors, represent the best agreement between theory and experiment known to us in all of radiation research. Both single and double strand breaks in SV40 DNA in EO buffer have recently been identified as 1-hit detectors. [18] This is a buffer which promotes the "indirect effect." Several E. coli mutants also act as 1-hit detectors. [19] Some interesting questions are raised by the differences in the characteristic $D_{37}$ doses for these mutants, as well as by their identification as 1-hit detectors. In biological systems the relative biological effectiveness is given by the expression $\mathrm{RBE}=\sigma E_{0} / L$, where $E_{0}$ is the characteristic $\left(\mathrm{D}_{37}\right)$ dose at which there is an average of 1 hit per target. 


\section{Physical and chemical 2 hit detectors}

It is inferred that supralinearity in TLD-100 is due to 2-hit trap structures. [20] For a parametric model, there is no conceptual difference between 2-hit trap, 2-hit response, and effects induced by adjacent pairs of electrons in "track interactions," between effects induced in the trapping stage or in the heating stage. By separating the 1-hit from the 2-hit response of TLD-100, it can be shown that the abrupt decline in response at high LET, different for different ions, is a consequence of "thin-down." [21] Two-hit responses have been found in radiation chemistry, in the production of $\mathrm{H}_{2} \mathrm{O}$ radicals in water, and in the production of $\mathrm{H}_{2}$ from benzene by energetic heavy ions. [22]

\section{Extension to Radiobiology}

Biological cells have complex structures. One seeks an oversimplified parametric model of minimal complexity, but which is capable of representing and extrapolating the experimental data. One may choose to think of a biological cell as somewhat like a "bean bag," in which there are many otherwise unidentified beans that are 1-hit targets within the nucleus of a cell, and that " $\mathrm{m}$ " of these must be activated to initiate the series of events leading to the expressed endpoint. For guidance in constructing the model, one calculates the cross section for a bean cluster whose response of the detector to gamma rays is taken to have the form of the m-target, 1-hit per target model of radiobiological target theory. As in all theoretical models, one makes an inductive leap-asserting that the observed cross section for cellular inactivation follows the form of the calculated response of our bean cluster, but has the size of the nucleus, because ions passing anywhere through the nucleus have the possibility of intersecting $\mathrm{m}$ targets. One must therefore introduce a fourth parameter to the three previously used for physical detectors, namely one representing the size of the bean bag, as well as one representing the size of the bean. This results in a four parameter model for radiobiological effects, in which the parameters-labeled $\mathrm{m}, \mathrm{K}, \mathrm{\sigma}_{0}$, and $E_{0}$ - are fitted to radiobiological data obtained with gamma rays and a series of bombardments with heavy ions through the response equations of the model. These equations have been given elsewhere [23] and will not be repeated here. Two new concepts, "ion-kill" and "gamma kill," are introduced, representing "intra-track" and "inter-track" interactions: ion-kill results from the interaction of cells with single ions, while gamma-kill results from interaction by cumulative damage from several ions, as it were in the gaps between inactivated cells along the ion's path. In the transition from low to high LET bombardments, there is a transition from gamma-kill with shouldered survival curves to ion-kill with exponential survival curves. The RBE for these cells attains a maximum value when about half the intersected cells are inactivated. Once the parameters of a particular cellular response have been evaluated, the response of a cell to an arbitrary radiation field may be calculated from track segment bombardments, provided only that the full photon-particleenergy spectrum of the field is known. This has been demonstrated for heavy ion beams and for $14 \mathrm{MeV}$ neutrons, and is currently being applied to space radiation effects. Cellular radiosensitivity parameters have been evaluated for cell killing for a variety of cells in vitro, for mutations and transformations in vitro, and even for lethal mutations in one organism, the nematode Caenorhabdifis elegans. For calculations in complex radiation environments there is now a reasonably good model of heavy ion penetration and fragmentation. [24]

There remain some problems with our biological model. Not all biological data fall into place, yet this is the most successful parametric model that has yet emerged. This model does not yet include biological repair, and there is controversy regarding the use of an m-target representation for extrapolation of gamma ray response to the lowest doses. Efforts are being made to resolve these questions.

\section{Acknowledgment}

This work is supported by the U.S. Department of Energy.

\section{References}

1. Katz, R., and Butts, J. J. Width of Ion and Monopole Tracks in Emulsion. Phys. Rev. 137: B198-B207 (1965)

2. Katz, R., and Kobetich, E. J. Formation of Etchable Tracks in Dielectrics. Phys. Rev. 170: 401-405 (1968)

3. Mazzei, R., and Bernaola, O. A. Track Experimental Data Related to Post-Irradiation Dynamic Processes. Nucl. Instrum. Methods Phys Res. B. (1992).

4. Butts, J. J., and Katz, R. Theory of RBE for Heavy Ion Bombardment of Dry Enzymes and Viruses. Radiat. Res. 30: 855-871 (1967).

5 Waligorski, M. P. R., Sinclair, G. L., and Katz, R. Inactivation of Dry Enzymes and Viruses by Energetic Heavy Ions. Radiat. Phys. Chem. 30: 201-208 (1988).

6. Katz, R., and Varma, M. N. Radial Distribution of Dose. In: Physical and Chemical Mechanisms in Radiation Biology, ed. W. A. Glass and M. N. Varma (New York: Plenum, 1991). 
7. Constantini, J. M., Brisard, F., Flament, J. L., Meftah, A., Toulemonde, M., and Hage-Ali, M. High Energy Heavy Ion Irradiation Damage in Yttrium Iron Garnet. Nucl. Instrum. Methods B (Proceedings of REI-6, 1992).

8. Katz, R., and Kobetich, E. J. Particle Tracks in Emulsion. Phys. Rev. 186: 344-351 (1969).

9. Jenson, M., Larsson, L., Mathiesen, O., and Rosander, R. Experimental and Theoretical Absorptance Profiles of Tracks of Fast Heavy Ions in Nuclear Emulsion. Phys. Scr. 13, 6574 (1976).

10. Katz, R., Li, A. S. F., Chang, Y. L., Rosman, R. L., and Benton, E. V. Tracks of Argon Ions in Ilford K-Series Nuclear Emulsions. In: Proc. 10th Intl. Conf. on Solid State Nuclear Track Detectors, Lyon, France, pp. 101-110 (Oxford: Pergamon, 1980).

11. Katz, R., and Kobetich, E. J. Response of NaI(Tl) to Energetic Heavy Ions. Phys. Rev. 170: 397-400 (1968).

12. Katz, R., Sinclair, G. L., and Waligorski, M. P. R. The Fricke Dosimeter as a 1-Hit Detector. Nuclear Tracks and Radiation Measurements 11: 301-307 (1986).

13. Bartlett, D. T., and Edwards, A. A., Investigations of the Dependence of Lyoluminescence Response on Radiation Quality and Its Relationship with the Gamma-Dose Response Function. In Proc. 6th Intl. Conf. on Solid State Dosimetry (1980).

14. Waligorski, M. P. R., Danialy, G., Loh, K. S., and Katz, R. Response of the Alanine Dosimeter to Charged Particle and Neutron Irradiations. Appl. Radiat. Isot. 40: 923-933 (1989).

15. Perez, A., Balanzat, E., and Dural, J. Experimental Study of Point-Defect Creation in High-Energy Heavy-Ion Tracks. Phys. Rev. B 41: 3943-3950 (1990).
16. Olsen, K. J., and Hansen, J. W. Experimental and Calculated Effectiveness of a Radiochromic Dye Film to Stopping $21 \mathrm{MeV}{ }^{7} \mathrm{Li}$ and $64 \mathrm{MeV}{ }^{16} \mathrm{O}$ Ions. Nucl. Instrum. Methods Phys. Res. B 5: 497-504 (1984).

17. Larsson, L., and Katz, R. Supralinearity of Thermoluminescent Dosimeters. Nucl. Instrum. Methods 138: 631-636 (1976).

18. Katz, R., and Wesely, S. Cross Sections for Single and Double Strand Breaks in SV-40 Virus in EO Buffer after HeavyIon Irradiation: Experiment and Theory. Radiat. Environ. Biophys. 30: 81-85 (1991).

19. Katz, R., and Zachariah, R. Experimental and Theoretical Cross Sections for E. coli Mutants B, B/r, and $\mathrm{B}_{\mathrm{s}-1}$ after Heavy-Ion Irradiation (forthcoming).

20. Waligorski, M. P. R., and Katz, R. Supralinearity of Peak 5 and Peak 6 in TLD-700. Nucl. Instrum. Methods 175: 48-50 (1980).

21. Luo, Daling, and Katz, R. Thindown in Lithium Fluoride. Nucl. Sci. Techn. (China) 2: 147-152 (1991).

22. Katz, R., and Huang, G-R. Track "Core" Effects in Heavy Ion Radiolysis. Radiat. Phys. Chem. 33: 345-349 (1989).

23. Katz, R., Sharma, S. C., and Homayoonfar, M. The Structure of Particle Tracks. In Topics in Radiation Dosimetry, Suppl. 1, ed. F. H. Attix (New York: Academic Press, 1972).

24. Shavers, M. R., Wilson, J. W., and Townsend, L. W. MultiGeneration Transport Theory as an Analytical Heavy Ion Transport Model. In Proc. Topical Meeting on New Horizons in Radiation Protection and Shielding, pp. 198-210 (Am. Nucl. Soc., 1992). 